\title{
End-to-End Delay Analysis in Cognitive Radio Ad Hoc Networks with Different Traffic Models
}

\author{
Jing Gao, ${ }^{1,2}$ Changchuan Yin, ${ }^{2}$ and Xi $\mathrm{Han}^{3}$ \\ ${ }^{1}$ School of Electronics and Communication Engineering, Tianjin Normal University, Tianjin 300387, China \\ ${ }^{2}$ Beijing Key Laboratory of Network System Architecture and Convergence, Beijing University of Posts and Telecommunications, \\ Beijing 100876, China \\ ${ }^{3}$ Information Network Center, Beijing University of Posts and Telecommunications, Beijing 100876, China
}

Correspondence should be addressed to Jing Gao; jing401@126.com

Received 8 June 2015; Accepted 12 July 2015

Academic Editor: Qilian Liang

Copyright (C 2015 Jing Gao et al. This is an open access article distributed under the Creative Commons Attribution License, which permits unrestricted use, distribution, and reproduction in any medium, provided the original work is properly cited.

\begin{abstract}
Delay and throughput are important metrics for network performance. We analyze the end-to-end delay of cognitive radio ad hoc networks for two traffic models: backlogged and geometric, respectively. By modelling the primary users as a Poisson point process and the secondary network deploying multihop transmissions, we derive the closed-form expression for the end-to-end delay in secondary networks. Furthermore, we optimize the end-to-end delay in terms of the hop number and the secondary transmission probability, respectively. The range of the optimal hop number and the equation satisfied by the optimal transmission probability are obtained for backlogged source models. The equation met by the optimal hop number is presented for geometric source models.
\end{abstract}

\section{Introduction}

With the rapid development of real-time transmissions in wireless communication networks, delay analysis has gained more and more attention in the literature. Compared with single-hop transmission, the analysis of multihop delay is more challenging. Since many factors can impact the end-toend delay in multihop networks, such as routing algorithm, network topology, traffic model, and data scheduling, and since it is unrealistic to analyze the end-to-end delay by taking all factors into consideration, we simplify the network topology as a "line network" by neglecting the routing algorithm to complete the analysis of end-to-end delay.

A "line network" consists of a source, many relays, and a destination with all relays being distributed along line from a source to its destination. So far, much research has been conducted on the performance of "line network." In $[1,2]$, end-to-end propagation speed is determined without the delay constraints with a channel model combined pathloss, fading with noise. Accordingly, the tradeoff between single-hop transmission and multihop transmission subject to an end-to-end delay constraint is studied in [3]. In [4], end-to-end packet delivery probability is derived based on the distances between neighboring nodes in a Poisson point process (PPP). In [5], "line network" is decomposed into many independent queues, and the end-to-end delay is determined by considering the combination of TDMA and ALOHA access protocol. All these works do not take the traffic style of the source node into consideration. With the increasing requirement for various types of traffic $[6,7]$ (such as document, video, and audio), it becomes important to characterize the network performance considering different types of traffic. In [8], the correlations between traffic statistics and channel qualities are investigated accompanied with their impact on the performance of multihop networks. In [9], the end-to-end delay is studied in Poisson network considering two traffic models: backlogged and geometric.

However, prior studies only focused on homogeneous networks without considering heterogeneous network model. A practical network usually consists of interdependent, interactive, and hierarchical network components which leads to a heterogeneous network structure. Different from [9], the purpose of this paper is to conduct a systematic study of the endto-end delay in cognitive radio (CR) ad hoc networks which is one of heterogeneous network models. In this paper, we evaluate the end-to-end delay of CR ad hoc networks in which 
the secondary nodes are assumed to be placed to form a line network. We derive the closed-form expression for the endto-end delay for backlogged and geometric arrival model of source traffic, respectively. Primary and secondary networks are supposed to be two PPPs which are independent of each other. Similar to that, in [9], waiting delay is considered by importing the node buffer accompanied with propagation delay. And the waiting delay of each node is modeled as a $\mathrm{M} / \mathrm{M} / 1$ model with infinite buffer.

The paper is organized as follows. Section 2 defines the system model and symbol notations. Section 3 analyzes the end-to-end delay of CR ad hoc network. Section 4 derives the end-to-end delay of secondary network for two traffic models. Section 5 presents the numerical results and some discussions. Finally, conclusions are drawn in Section 6.

\section{System Model}

We consider a scenario where primary users and secondary users coexist in the same two-dimensional plane. Secondary users employ underlay spectrum sharing method to access the licensed channel, that is, transmit their packets while keeping the quality of service (QoS) of primary network. In the following, we will define the primary and secondary network models, respectively.

2.1. Primary Network Model. Assume that the locations of primary transmitters follow a PPP $\Phi_{\mathrm{PT}}$ with density $\lambda_{P}$. Each primary receiver associates with one designated primary transmitter with $R_{P}$ distant away. According to the displacement theorem [10], the locations of the primary receivers formulate another $\mathrm{PPP} \Phi_{\mathrm{PR}}$ with density $\lambda_{P}$. All transmitters are assumed to send their packets with the same power $\sigma_{P}$. Signals undergo path-loss and small scale Rayleigh fading so that the power caught by a receiver is $\sigma_{P} h R_{P}^{-\alpha}$, where $h$ is the small scale fading coefficient having exponential distribution with mean of 1 and $\alpha>2$ is the path-loss factor.

\subsection{Secondary Network Model}

2.2.1. Topology. Secondary network is composed of many multihop paths, each of which comprises a source user, $N-1$ relay users, and a destination user. The distance from a source user to its destination is $R$. Figure 1 is the topology of a twohop network. Supposing that all source users are distributed as a PPP with density $\lambda_{S}$, then all relay users formulate a PPP with density $(N+1) \lambda_{s}$. All relay users are equidistantly distributed on the line from source user to the destination user; one hop distance is $R / N$. Index $n=0, \ldots, N$ as the source, the relay, and destination users. The power of all transmitters is assumed to be the same and is represented by $\sigma_{S}$. Time is divided into slots and all users are synchronized to one clock. We assume no space reuse on one path; that is, users along one path are not allowed to send packets in the same time slot. Therefore, the locations of secondary transmitters in each slot follow a PPP with density $\lambda_{S}$. Each user has an infinite buffer in which data is queued by FIFO (first-in-first-out) mode.

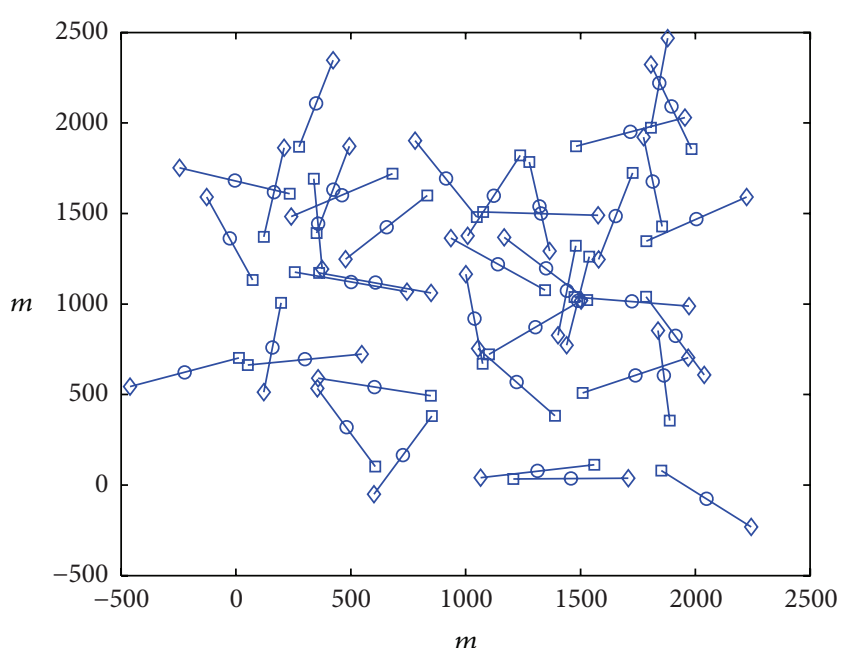

$\square$ Source
$\bigcirc$ Relay
$\diamond$ Destination

FIgUre 1: Topology of a two-hop network.

2.2.2. Traffic Model. In data networks, there are many types of traffic, such as video, audio, and files. Different traffic model has different impacts on the network performance. Similar to that in [9], we consider the end-to-end delay for two traffic models in this paper: backlogged and geometric:

(i) Backlogged sources: source users always have data packets to send.

(ii) Geometric sources: data packets arrive at the source user with probability $a$ each $N$ time slots.

2.2.3. MAC Protocol. A combination of TDMA and ALOHA protocol is adopted. Since time is divided into slots, source, relay, and destination users send their packets in turn at the beginning of each slot. The source and relay users will send their packets when they are permitted to transmit, with probabilities $p_{o}$ and $p$, respectively. Packets are successfully received at the time slot if signal-to-interference ratio (SIR) caught by their receivers is greater than the threshold $\beta$. Otherwise, the source and relay users will receive a negative feedback and the packets are still put first in the queue for retransmission.

Take a two-hop transmission with backlogged sources; for example, set $t=0$ when the source user of a path begins to send packets with probability $p_{o}$. If the SIR at the relay user is greater than $\beta$, an ACK will be sent to the source, which indicates that the packets are successfully received by the relay, and the packets will be discarded from the queue of the source. Otherwise, the packets are still at the head of the queue in the source. At the beginning of the next time slot, the relay users will transmit their packets with probability $p$. Similarly, the destination will send an ACK to the relay if it successfully received the packets. And the relay discards the current packets from its queue according to the ACK. At the beginning of the next time slot, the sources 
transmit their packets in the queue again and so on. In this paper, we ignore the ambient thermal noise and consider the interference coming from both the secondary transmitters and the primary transmitters, which is different from [9].

2.3. SIR Based Successful Transmission. We consider the wireless channel which undergoes both large scale path-loss and small scale Rayleigh fading. Power gain of the channel in both networks is given by

$$
G(r)=h r^{-\alpha}
$$

where $r$ is the link distance.

(i) We consider a typical primary receiver $y$ on the origin; its transmitter $s$ can successfully send the packets to it if and only if

$$
\operatorname{SIR}_{y}=\frac{\sigma_{P} h R_{P}^{-\alpha}}{I} \geqslant \beta,
$$

where $I$ is the sum of interference coming from other concurrent transmitters.

(ii) Considering a certain secondary user $n$ along an arbitrary path accessing the channel at time $t$, its next hop user $n+1$ could successfully receive the packets from user $n$ if and only if

$$
\operatorname{SIR}_{n+1}=\frac{\sigma_{S} h(R / N)^{-\alpha}}{I^{\prime}(t)} \geqslant \beta_{1}
$$

where $I^{\prime}(t)$ is the sum of interference of other transmitters at time $t$.

\section{Successful Transmission Probability}

Successful transmission probability is the probability that a receiver could receive the packets successfully from its transmitter in a time slot.

\subsection{Successful Transmission Probability of Standalone Primary} Network. When the secondary network is absent, based on the SIR transmission criterion, the successful transmission probability of a typical primary transmitter $x_{0}$ is given by

$$
P_{\mathrm{SUC}}^{1}=\operatorname{Pr}\left(\operatorname{SIR}_{1} \geqslant \beta\right)=\operatorname{Pr}\left(\frac{\sigma_{P} h R_{P}^{-\alpha}}{I_{P}} \geqslant \beta\right) \geqslant 1-\varepsilon_{0},
$$

where $I_{P}=\sum_{x \in \Phi_{\mathrm{PT}} \backslash x_{0}} \sigma_{P} h|x|^{-\alpha}$ is the sum of interference power of other primary transmitters. $\varepsilon_{0}$ is the outage constraint of the primary network.

According to Lemma 1 in [11], we have

$$
P_{\mathrm{SUC}}^{1}=\operatorname{Pr}\left(h \geqslant \sigma_{P}^{-1} \beta I_{P} R_{P}^{\alpha}\right)=e^{-\sigma_{P}^{-1} \beta I_{P} R_{P}^{\alpha}}=e^{-\lambda_{P} \beta^{2 / \alpha} C_{\alpha} R_{P}^{2}},
$$

where $C_{\alpha}=2 \pi^{2} / \alpha \sin (2 \pi / \alpha)$. Putting (5) into (4), we obtain the density range of the primary transmitters as

$$
0<\lambda_{P} \leqslant \lambda_{P, m}=\frac{-\ln \left(1-\varepsilon_{0}\right)}{C_{\alpha} \beta^{2 / \alpha} R_{P}^{2}} .
$$

3.2. Successful Transmission Probability of the Secondary Network with Backlogged Sources. When the secondary network is presented, the successful transmission probability of both primary and secondary networks is closely related to the density of the secondary transmitters due to the interference they introduced. Supposing secondary user $k$ accesses the channel at $t=0$, for backlogged traffic sources, the distribution of secondary transmitters in each time slot is characterized in Lemma 1 as follows.

Lemma 1. For backlogged sources and $p_{o}<p$, secondary transmitters follow a PPP $\Phi_{S T}(t)$ with density $\lambda_{S} p_{o}$ within any time slot $\left(t, t+T_{s}\right]$.

Proof. Considering that secondary transmitters send their packets independently and have the same successful transmission probability $p_{s}$, the queue of a relay user can reach a steady state if and only if $p_{o} p_{s}<p p_{s}$; that is, $p_{o}<p$. Under the steady state circumstance, we discuss the distribution of secondary transmitters in the following two cases:

(i) Time slots begin at $t=0$ or $\bmod (t+1, N)=0$ : in these time slots, secondary source users transmit their packets with probability $p_{o}$. Since source users are conformed to a PPP with density $\lambda_{S}$ in each time slot, therefore, the distribution of secondary transmitters is a PPP with density $\lambda_{S} p_{o}$.

(ii) Time slots begin at any other $t$. In these slots, relay users are allowed to send their packets. Unlike source users, relay users will transmit with a certain probability which is the product of the probability of nonempty queue and the probability of accessing channel. According to M/M/1 queue model, the probability that the queue of a relay buffer is nonempty is $p_{o} p_{s} / p p_{s}=p_{o} / p$. Therefore, the probability of relay users sending their packets is $\rho=p_{o} / p \cdot p=p_{o}$. Since there is no intraroute interference along each multihop route and the relay users send their packets in turn, as such secondary transmitters in these time slots follow a PPP with density $\lambda_{S} P_{o}$.

In conclusion, whenever the source users or the relay users are permitted to send packets, the distribution of the secondary transmitters follows a PPP with density $\lambda_{S} p_{o}$.

This completes the proof.

According to Lemma 1, the successful transmission probability of the primary network is

$$
\begin{aligned}
\mathrm{P}_{\mathrm{SUC}}^{2} & =\operatorname{Pr}\left(\operatorname{SIR}_{2} \geqslant \beta\right)=\operatorname{Pr}\left(\frac{\sigma_{P} h R_{P}^{-\alpha}}{I_{P}+I_{S}} \geqslant \beta\right) \\
& =\psi_{I_{P}}\left(\sigma_{P}^{-1} \beta R_{P}^{\alpha}\right) \psi_{I_{S}}\left(\sigma_{P}^{-1} \beta R_{P}^{\alpha}\right),
\end{aligned}
$$

where $\psi_{I}(i)$ is the Laplace transformation of the probability density function (PDF) and

$$
\begin{aligned}
& \psi_{I_{P}}(i)=\exp \left(-C_{\alpha} \lambda_{P}\left(\sigma_{P} i\right)^{2 / \alpha}\right), \\
& \psi_{I_{S}}(i)=\exp \left(-C_{\alpha} \lambda_{S} p_{o}\left(\sigma_{S} i\right)^{2 / \alpha}\right) .
\end{aligned}
$$


Substituting $i=\sigma_{P}^{-1} \beta R_{P}^{\alpha}$ into (8) and putting these results into (7), we have

$$
P_{\mathrm{SUC}}^{2}=\exp \left(-C_{\alpha} \beta^{2 / \alpha} R_{P}^{2}\left(\lambda_{P}+\lambda_{S} p_{o}\left(\frac{\sigma_{S}}{\sigma_{P}}\right)^{2 / \alpha}\right)\right) .
$$

Since the outage constraint of primary network is $\varepsilon_{0}, P_{\mathrm{SUC}}^{2}$ must satisfy $P_{\mathrm{SUC}}^{2} \geqslant 1-\varepsilon_{0}$. Putting $P_{\mathrm{SUC}}^{2}$ into the inequality, we solve the density range of secondary users for backlogged sources as

$$
0<\lambda_{S} \leqslant \lambda_{S, m 1}=\frac{1}{p_{o}}\left(\frac{\sigma_{P}}{\sigma_{S}}\right)^{2 / \alpha}\left(\lambda_{P, m}-\lambda_{P}\right) .
$$

All $\lambda_{S}$ in the following analysis must meet the limit in (10). Supposing that each user in a multihop path sends packets with probability $p$ independently, we derive the successful transmission probability $p_{s}$ for one single hop in the following lemma.

Lemma 2. Considering the given MAC protocol, the successful transmission probability for one single hop is given by

$$
p_{s 1}=p_{s m} p_{s s}
$$

where

$$
p_{s m}=\exp \left(-\lambda_{P} C_{\alpha}(\delta)^{2 / \alpha}\left(\frac{R}{N}\right)^{2}\right)
$$

with $\delta=\beta_{1} \sigma_{S} / \sigma_{P}$,

$$
p_{s s}=\exp \left(-\lambda_{s} p_{o} C_{\alpha} \beta_{1}^{2 / \alpha}\left(\frac{R}{N}\right)^{2}\right) .
$$

Proof. The successful transmission probability for one single hop along a multihop path for secondary network is

$$
p_{s 1}=\operatorname{Pr}\left(\operatorname{SIR}_{S}>\beta_{1}\right),
$$

where $\beta_{1}$ is a predetermined threshold. Since there is no intrainterference but there is interinterference in secondary network, we have

$$
\begin{aligned}
p_{s 1} & =\operatorname{Pr}\left(\frac{\sigma_{S} h(R / N)^{-\alpha}}{I_{P}+I_{S}} \geqslant \beta_{1}\right) \\
& =e^{-\lambda_{P} C_{\alpha}(\delta)^{2 / \alpha}(R / N)^{2}} e^{-\lambda_{S} p_{o} C_{\alpha} \beta_{1}^{2 / \alpha}(R / N)^{2}}=p_{s m} \cdot p_{s s} .
\end{aligned}
$$

This completes the proof.

\subsection{Successful Transmission Probability of the Secondary Network for Geometric Sources}

Lemma 3. Supposing the packets arrive at the source users every $N$ slots with probability $a$, if $a<p_{s m} e^{-1} \min \left(p, m^{-1}\right)$, then the successful transmission probability of secondary network is

$$
p_{s 2}=p_{s m} e^{W\left(-m a / p_{s m}\right)}
$$

and the probability of a secondary user to be a transmitter is

$$
\rho=\frac{a e^{W\left(-m a / p_{s m}\right)}}{p_{s m}},
$$

where $p_{\text {sm }}=e^{-\lambda_{P} C_{\alpha}\left(\beta_{1} \delta\right)^{2 / \alpha}(R / N)^{2}}, m=\lambda_{S} C_{\alpha} \beta_{1}^{2 / \alpha}(R / N)^{2}$ and $W(\cdot)$ is Lambert $W$ function (Lambert $W$ function is the unique solution of the function $\left.z=x e^{x}\right)$.

Proof. A queue can reach steady state if and only if $a<$ $p_{s m} e^{-1} \min \left(p, m^{-1}\right)$. In this mode, the probability of a packet arriving at a relay user is $a$. Thus, a secondary user could be transmissions at a probability

$$
\rho=\frac{a}{p p_{s 2}} \cdot p=\frac{a}{p_{s 2}} .
$$

And the successful probability of a single-hop transmission is

$$
p_{s 2}=\operatorname{Pr}\left(\frac{\sigma_{S} h(R / N)^{-\alpha}}{I_{P}+I_{S 2}} \geqslant \beta_{1}\right) \text {. }
$$

Combined with the result in (15), we have

$$
\begin{aligned}
p_{s 2} & =e^{-\lambda_{P} C_{\alpha}(\delta)^{2 / \alpha}(R / N)^{2}} e^{-\lambda_{S} a C_{\alpha} \beta_{1}^{2 / \alpha}(R / N)^{2} / p_{s 2}} \\
& =p_{s m} e^{-m a / p_{s 2}} .
\end{aligned}
$$

Rewrite (20) as

$$
-\frac{m a}{p_{s 2}} \exp \left(-\frac{m a}{p_{s 2}}\right)=-\frac{m a}{p_{s m}}
$$

or

$$
-\frac{m a}{p_{s 2}}=W\left(-\frac{m a}{p_{s m}}\right)
$$

Substituting (22) into (20) and (18), respectively, we derive the results in (16) and (17).

In addition, $W\left(-m a / p_{s m}\right)$ has unique solution when $-m a / p_{s m} \geqslant-e^{-1}$. Combined with the condition of a queue reaching a steady state, we solve the following inequality and obtain the range of $a$ as given in Lemma 3:

$$
\begin{aligned}
-\frac{m a}{p_{s m}} & \geqslant-e^{-1}, \\
a & <p p_{s m} e^{W\left(-m a / p_{s m}\right)} .
\end{aligned}
$$

The proof is completed.

The density of the secondary users is further limited by the following lemma. 
Lemma 4. Supposing that the packets arrive at the source users every $N$ slots with probability $a$, the density of the secondary source users is bounded by

$$
\lambda_{S}<\lambda_{S, m 2}=\frac{p_{s m} \delta^{2 / \alpha}\left(-\lambda_{P} c_{1}-\ln \left(1-\varepsilon_{0}\right)\right)}{a c_{1}},
$$

where $c_{1}=C_{\alpha} \beta^{2 / \alpha} R_{P}^{2}$.

Proof. Being overlaid with secondary network for geometric sources, the successful transmission probability of the primary network is

$$
P_{\mathrm{SUC}}^{22}=e^{-C_{\alpha} \beta^{2 / \alpha} R_{P}^{2}\left(\lambda_{P}+\lambda_{S} \rho\left(\sigma_{S} / \sigma_{P}\right)^{2 / \alpha}\right)} .
$$

Let $c_{1}=C_{\alpha} \beta^{2 / \alpha} R_{P}^{2}, c_{2}=C_{\alpha} \beta_{1}^{2 / \alpha}(R / N)^{2}$; substitute $\rho$ in (17) into (25); we have

$$
P_{\mathrm{SUC}}^{22}=e^{-\lambda_{P} c_{1}} e^{-a \delta^{-2 / \alpha} \lambda_{S} c_{1} / p_{s m} e^{-W\left(-\lambda_{S} c_{2} a / p_{s m}\right)}} \geqslant 1-\varepsilon_{0} .
$$

Take logarithm at both sides of the inequality above; we have

$$
\lambda_{S} e^{-W\left(-\lambda_{S} a c_{2} / p_{s m}\right)} \leqslant \frac{p_{s m} \delta^{2 / \alpha}\left(-\lambda_{P} c_{1}-\ln \left(1-\varepsilon_{0}\right)\right)}{a c_{1}} .
$$

Since $e^{-W\left(-\lambda_{S} a c_{2} / p_{s m}\right)}>1$, we have $\lambda_{S}<\lambda_{S, m 2}=$ $p_{s m} \delta^{2 / \alpha}\left(-\lambda_{P} c_{1}-\ln \left(1-\varepsilon_{0}\right)\right) / a c_{1}$.

This completes the proof.

\section{Delay Analysis of CR Ad Hoc Networks}

In this section, we assume the types of data transmission for each hop along the multihop route are independent of each other and study the end-to-end delay of CR ad hoc networks in a way similar to that in [5].

\subsection{The End-to-End Delay of Secondary Network for \\ Backlogged Sources}

Theorem 5. In the case of backlogged sources, the end-to-end delay of an N-hop path in the secondary network is given by

$$
D_{e 2 e}^{(1)}=\frac{N}{p_{o} p_{s 1}}+\frac{N(N-1)\left(1-p p_{s 1}\right)}{p_{s 1}\left(p-p_{o}\right)} .
$$

Proof. The end-to-end delay is the sum of delays at source user and relays on a path. According to the TDMA and ALOHA combined MAC protocol, the propagation time from the source user to its relay is easyly obtained as follows:

$$
D_{s}=N\left(\frac{1}{p_{o} p_{s 1}}-1\right)+1 \text {. }
$$

For relay users, the waiting time is also needed to be calculated. Since all users are assumed to transmit their packets independently, we only need to calculate the waiting time for one single relay and then produce it with $(N-1)$ to obtain the total waiting time along the multihop route.
Referring to the propagation time for source user, we have the propagation time for a relay as

$$
D_{r s}=N\left(\frac{1}{p p_{s 1}}-1\right)+1
$$

Take the first relay user, for example; the nonempty probability of its queue is $P_{0}=1-p_{o} p_{s 1} / p p_{s 1}=1-p_{o} / p$. The probability of the relay user to be in $k$ state is

$$
P_{k}=\frac{q_{01} q_{12}^{k-1}}{q_{10}^{k}} P_{0}=\left(\frac{p_{o}}{p}\right)^{k}\left(1-\frac{p_{o}}{p}\right) \frac{\left(1-p p_{s 1}\right)^{k-1}}{\left(1-p_{o} p_{s 1}\right)^{k}} .
$$

Based on Little's theorem [12], the queue length for steady state $L_{a}$ is

$$
L_{a}=\sum_{k=2}^{\infty}(k-1) P_{k}=\frac{p_{o}^{2}\left(1-p p_{s 1}\right)}{p\left(p-p_{o}\right)} .
$$

Since the average length of a queue equalling the data arrival rate produces the mean waiting time [12], the mean waiting time in a queue is

$$
D_{r w}=\frac{L_{a} N}{p_{o} p_{s 1}}=\frac{N p_{o}\left(1-p p_{s 1}\right)}{p p_{s 1}\left(p-p_{o}\right)} .
$$

And the end-to-end delay is

$$
D_{e 2 e}^{(1)}=D_{s}+(N-1)\left(D_{r s}+D_{r w}\right) .
$$

Substituting $D_{s}, D_{r s}$, and $D_{r w}$ into (34), we obtain the result in (28).

This completes the proof.

4.2. The End-to-End Delay of Secondary Network for Geometric Sources. For a S-D multihop path, the probability of a packet arriving at each user (including source user and relay user) is $a$. The probability of successful transmission is $p p_{s 2}$. For simple calculation, we derive the upper bound of the end-to-end delay, which starts being calculated from the data packets arriving at the tail of the queue of a source user. And the end-to-end delay is the sum of propagating time and waiting time of $N$ users:

$$
\bar{D}_{e 2 e}^{(2)}=N\left(D_{r s}^{(2)}+D_{r w}^{(2)}\right)
$$

According to Theorem 5, the propagating and the waiting time for each user could be obtained as follows:

$$
\begin{aligned}
& D_{r s}^{(2)}=N\left(\frac{1}{p p_{s 2}}-1\right)+1, \\
& D_{r w}^{(2)}=\frac{N a\left(1-p p_{s 2}\right)}{p p_{s 2}\left(p p_{s 2}-a\right)},
\end{aligned}
$$


respectively. Therefore, the upper bound of the end-to-end delay is

$$
\begin{aligned}
\bar{D}_{e 2 e}^{(2)} & =N\left(D_{r s}^{(2)}+D_{r w}^{(2)}\right) \\
& =N^{2}\left(\frac{1}{p p_{s 2}}-1\right)+N+\frac{N^{2} a\left(1-p p_{s 2}\right)}{p p_{s 2}\left(p p_{s 2}-a\right)} \\
& =\frac{N^{2}\left(1-p p_{s 2}\right)}{p p_{s 2}-a}+N .
\end{aligned}
$$

Summing the delay of the source user, we have the end-to-end delay as

$$
D_{e 2 e}^{(2)}=\frac{N^{2} p p_{s 2}\left(1-p p_{s 2}\right)+N p p_{s 2}-a N}{p p_{s 2}\left(p p_{s 2}-a\right)} .
$$

4.3. Optimization of the End-to-End Delay. In the following, we will optimize the end-to-end delay for backlogged and geometric sources in terms of $N$ and $p_{o}$.
Theorem 6. For backlogged source traffic model, the end-toend delay has an upper bound $\bar{D}_{e 2 e}^{(1)}$, and

$$
\bar{D}_{e 2 e}^{(1)}=\frac{N}{p_{o} p_{s 1}}+\frac{N(N-1)}{p_{s 1}\left(p-p_{o}\right)} .
$$

Proof. For $0<p<1$ and $0<p_{s 1}<1$, it is obvious that $1-p p_{s 1}<1$. While $p \rightarrow 0, \bar{D}_{e 2 e}^{(1)}$ is the tight upper bound of the end-to-end delay $D_{e 2 e}^{(1)}$, which completes the proof.

Compared with $D_{e 2 e}^{(1)}, \bar{D}_{e 2 e}^{(1)}$ concludes the waiting time in the queue of the source user which has nothing to do with the hop number $N$. Therefore, it is reasonable for us to optimize $\bar{D}_{e 2 e}^{(1)}$ instead of $D_{e 2 e}^{(1)}$ in terms of hop number $N$.

Proposition 7. For backlogged sources, the optimal hop number to minimize the upper bound of the end-to-end delay is

$$
N^{*}= \begin{cases}{\left[\sqrt{C_{\alpha}\left(\lambda_{P}(\delta)^{2 / \alpha}+\lambda_{S} p_{o} \beta_{1}^{2 / \alpha}\right)} R, \sqrt{2 C_{\alpha}\left(\lambda_{P}(\delta)^{2 / \alpha}+\lambda_{S} p_{o} \beta_{1}^{2 / \alpha}\right)} R\right),} & p_{o} \in\left(0, \frac{p}{2}\right], \\ {\left[1, \sqrt{C_{\alpha}\left(\lambda_{P}(\delta)^{2 / \alpha}+\lambda_{S} p_{o} \beta_{1}^{2 / \alpha}\right)} R\right) .} & p_{o} \in\left(\frac{p}{2}, p\right) .\end{cases}
$$

The optimal transmission probability meets the equation in the following:

$$
\begin{gathered}
\left(m p_{o}^{*}-1\right)\left[\left(p-p_{o}^{*}\right)^{2}-(N-1)\left(p_{o}^{*}\right)^{2}\right] \\
+(N-1) m p\left(p_{o}^{*}\right)^{2}=0 .
\end{gathered}
$$

Proof. Take the derivative of $\bar{D}_{e 2 e}^{(1)}$ with respect to $N$; we obtain

$$
\begin{aligned}
& \frac{\partial \bar{D}_{e 2 e}^{(1)}}{\partial N} \\
& =\frac{2 N^{3}+\left(p / p_{o}-2\right) N^{2}-2 c_{3} R^{2} N+4 c_{3} R^{2}-2 c_{3} R^{2} p / p_{o}}{p_{s 1}\left(p-p_{o}\right)},
\end{aligned}
$$

where $c_{3}=C_{\alpha}\left(\lambda_{P}(\delta)^{2 / \alpha}+\lambda_{S} p_{o} \beta_{1}^{2 / \alpha}\right)$. Since $p_{o}<p$ is the prerequisite for a queue entering steady state; that is, $p-p_{o}>$ 0 , Therefore, the sign of (43) is determined by $f(N)$, and

$$
\begin{aligned}
f(N)= & 2 N^{3}+\left(\frac{p}{p_{o}}-2\right) N^{2}-2 c_{3} R^{2} N+4 c_{3} R^{2} \\
& -\frac{2 c_{3} R^{2} p}{p_{o}} \\
= & \left(\frac{p}{p_{o}}-2\right)\left(N^{2}-2 c_{3} R^{2}\right)+2 N\left(N^{2}-c_{3} R^{2}\right) .
\end{aligned}
$$

In the following, we discuss the sign of $f(N)$ in two cases:

(1) While $0<p_{o} \leqslant p / 2$,

$$
\begin{aligned}
& \text { if } N^{2}>2 c_{3} R^{2} \text {, then } N>\sqrt{2 c_{3}} R \text {, and } f(N)>0 \text {; } \\
& \text { if } N^{2}<2 c_{3} R^{2} \text {, then } N<\sqrt{2 c_{3}} R \text {, and } f(N)<0 \text {; } \\
& \text { if } p_{o}=p / 2, N^{2}=c_{3} R^{2} \text {, then } f(N)=0 \text {. }
\end{aligned}
$$

The optimal hop number is

$$
N^{*}=\sqrt{c_{3}} R=\sqrt{C_{\alpha}\left(\lambda_{P}(\delta)^{2 / \alpha}+\lambda_{S} p_{o} \beta_{1}^{2 / \alpha}\right)} R .
$$

(2) While $p / 2<p_{o}<p$, let $N=\sqrt{k c_{3}} R, k \geqslant 1$, and we obtain

$$
\begin{aligned}
\frac{f(N)}{c_{3} R^{2}} & =\left(\frac{p}{p_{o}}-2\right)(k-2)+2 \sqrt{k c_{3}} R(k-1) \\
& >-(k-2)+2 \sqrt{k c_{3}} R(k-1) \\
& >\left(2 \sqrt{k c_{3}} R-1\right)(k-1) \geqslant 0 .
\end{aligned}
$$

Since $k \geqslant 1, N \geqslant \sqrt{c_{3}} R$.

Let $\partial \bar{D}_{e 2 e}^{(1)} / \partial p_{o}=0$, we derive the optimal transmission probability for the source user as shown in the proposition.

The proof is completed. 
Proposition 8. If the packets arrive at the source user in a probability a each $N$ time slot, we optimize $\bar{D}_{e 2 e}^{(2)}$ instead of $D_{e 2 e}^{(2)}$ in the following:

$$
\bar{D}_{e 2 e}^{(2)}=\frac{N^{2}\left(1-p p_{s 2}\right)}{p p_{s 2}-a}+N .
$$

And the optimal hop number $N^{*}$ to minimize the end-to-end delay should meet the expression as follows:

$$
\begin{aligned}
2(1- & \left.p p_{s m}^{*} e^{W^{*}}\right)\left(p p_{s m}^{*} e^{W^{*}}-a\right)\left(N^{*}\right)^{2} \\
& +\left(p p_{s m}^{*} e^{W^{*}}-a\right)^{2} N^{*} \\
& +\frac{2 p p_{s m}^{*} e^{W^{*}}(1-a)\left(k_{2} W^{*}+k_{1} m^{*}\right)}{m^{*}\left(1+W^{*}\right)}=0
\end{aligned}
$$

where $k_{1}=\lambda_{P} C_{\alpha}(\delta)^{2 / \alpha} R^{2}, k_{2}=\lambda_{S} C_{\alpha} \beta_{1}^{2 / \alpha} R^{2}, p_{s m}^{*}=$ $\exp \left(-k_{1}\left(N^{*}\right)^{-2}\right), m^{*}=k_{2}\left(N^{*}\right)^{-2}$, and $W^{*}=W\left(-m^{*} a / p_{s m}^{*}\right)$.

The proof is similar to that of Proposition 7 and therefore is omitted.

\section{Numerical Results and Discussion}

In this section, we present some numerical results based on the above theoretical analysis. Unless otherwise specified, the network parameters are set as follows: $\beta=3 \mathrm{~dB}, \beta_{1}=1 \mathrm{~dB}$, $\varepsilon_{0}=0.05, \alpha=4, R_{P}=20 \mathrm{~m}, R=100 \mathrm{~m}, p_{o}=0.1$, and $p=0.2$. Putting these parameters into (5), (9), and (24) and letting $\lambda_{P}=1 \times 10^{-5}$, we obtain $\lambda_{P, m}=1.84 \times 10^{-5}, \lambda_{S, m 1}=$ $1.2 \times 10^{-4}$, and $\lambda_{S, m 2}=7.42 \times 10^{-5}$.

In Figure 2, we illustrate $D_{e 2 e}^{(1)}$ in (28) versus density of primary users $\lambda_{P}$ for backlogged source model. As shown in the figure, we see that $D_{e 2 e}^{(1)}$ increases linearly with $\lambda_{P}$. The reason is that, on the one hand, the increasing $\lambda_{P}$ leads to more interference from the primary users and, on the other hand, this also decreases the maximum allowable density of the secondary users which decreases the intrainterference. Since the effect of increasing of interinterference is larger than that of the reducing of the intrainterference, the probability of successful transmission for the secondary network becomes smaller which increases the end-to-end delay. Therefore, the density of the primary users plays a significant role in the delay performance of the secondary network.

In Figure 3, we show the end-to-end delay versus the density of the secondary source users for geometric sources with varied $\lambda_{P}$. Given $\lambda_{P}=1 \times 10^{-5}, a=0.05$, we derive $\lambda_{S, m 2}=2.346 \times 10^{-4}$. It is shown that the end-to-end delay increases monotonically with increasing $\lambda_{S}$ in range $(0,1 \times$ $\left.10^{-4}\right)$. This explains that increased density of primary users will increase the interinterference and thus increase the endto-end delay of the secondary network.

In Figure 4, we show the end-to-end delay for backlogged sources versus the optimal hop number $N^{*}$ when $\lambda_{S}=1 \times$ $10^{-4}$. It is shown that the optimal hop number $N^{*}=3$ when $p_{o}=0.05$ and $N^{*}=4$ when $p_{o}=0.15$. It is worth noting

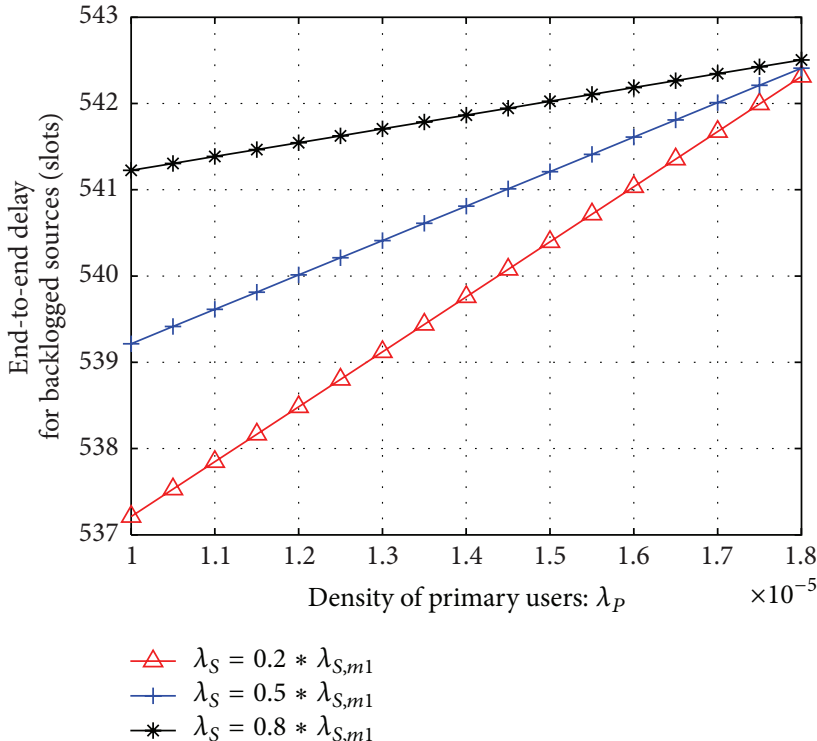

FIGURE 2: End-to-end delay versus density of primary users for backlogged source model.

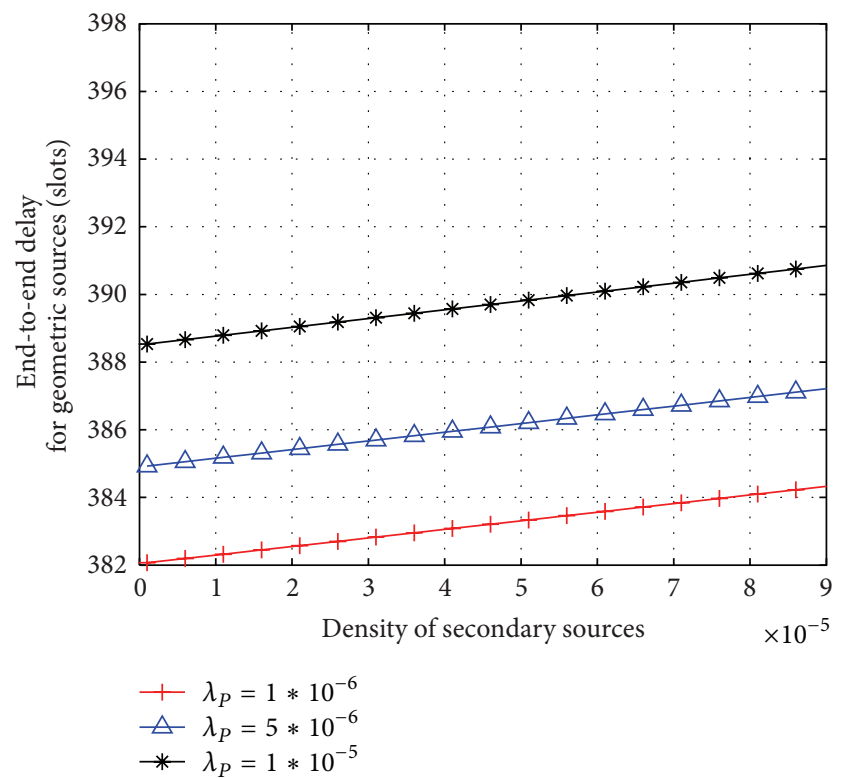

FIGURE 3: End-to-end delay versus density of secondary source users for geometric source model.

that higher transmission power of source user causes more intrainterference. In this case, we can reduce the hop distance to obtain maximized successful transmission probability and then the minimized end-to-end delay. Similarly, the higher the density of the primary transmitters, the larger the optimal hop number.

In Figure 5, we draw the end-to-end delay with backlogged sources versus transmission probability $p_{o}$ of the source users. Since the necessary condition for node queue entering steady state is $p_{o}<p$. It is shown that there is an optimal $p_{o}$ in the range of $(0, p)$ to minimize the end-to-end 


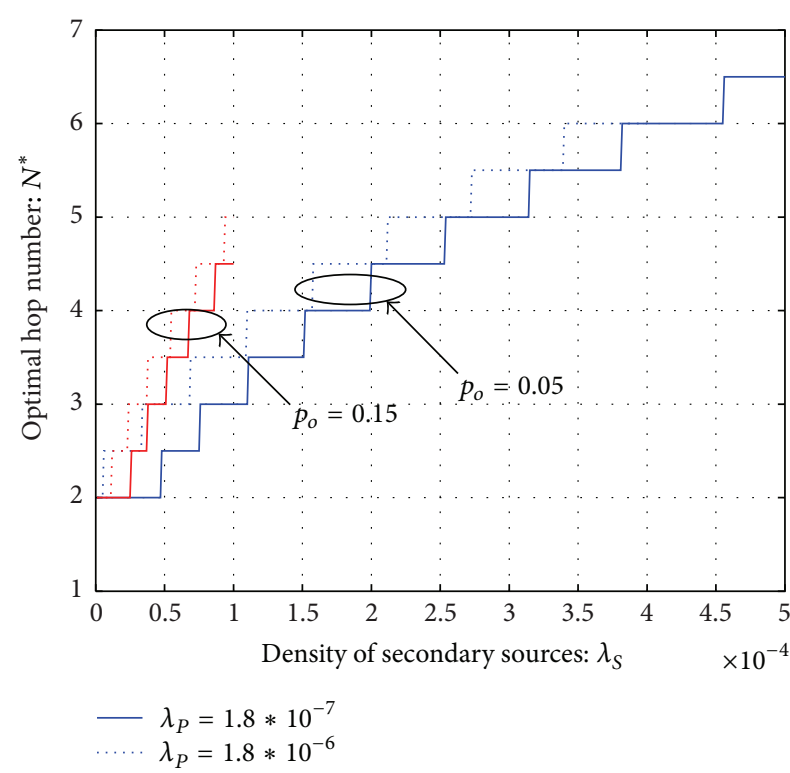

FIGURE 4: Optimal hop number versus density of secondary source users.

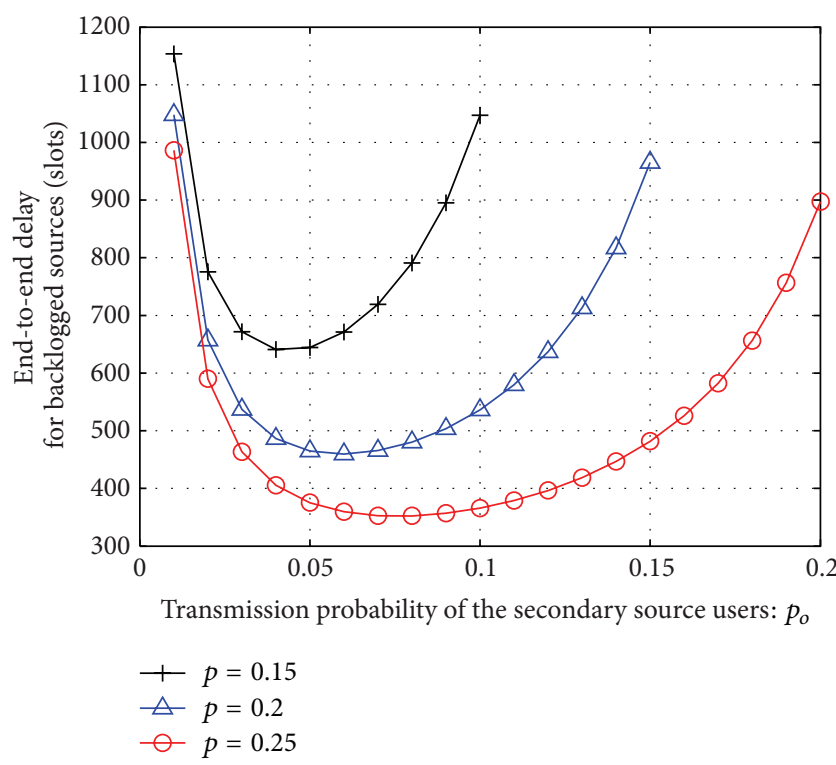

FIGURE 5: End-to-end delay for backlogged sources versus transmission probability of the secondary source users.

delay. When $p=0.15$, putting the parameters into (42), we solve the optimal transmission probability of the source users as $p^{*}=0.041$ which agrees with the numerical results shown in the figure.

In Figure 6, we present the end-to-end delay for geometric sources versus hop number $N$. It is seen that there is an optimal hop number $N^{*}$ to minimize the end-to-end delay no matter how the access probability of the relay users vary with $p$. In addition, the larger the $p$, the lower the delay. This is because when the access probability becomes larger, the waiting delay of each relay becomes smaller which leads to lower delay.

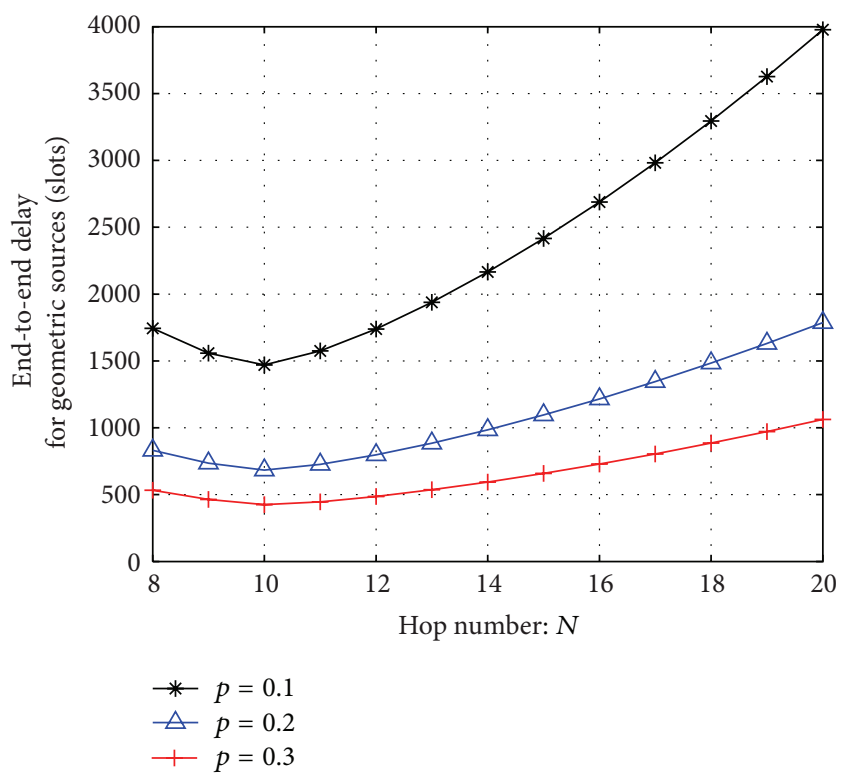

Figure 6: End-to-end delay for backlogged sources versus hop number.

\section{Conclusions}

We studied the end-to-end delay in CR ad hoc networks for two traffic sources models: backlogged and geometric. First, we modelled the primary users as PPP and the secondary users opportunistically access the channel with a combination of TDMA with ALOHA protocol. Secondly, we derived the successful transmission probability for both the primary and the secondary networks, respectively. Thirdly, we obtained the closed-form expression for the end-to-end delay with the above two traffic models, respectively. Finally, by optimizing the end-to-end delay, we obtained the range of the optimal hop number for backlogged sources and we also present an equation with which the optimal hop number should satisfy geometric sources. The numerical results showed that (1) due to the outage constraints of the primary network, an optimal hop number and transmission probability for source user could be derived to minimize the end-to-end delay for backlogged sources and (2) an optimal hop number can be got to minimize the end-to-end delay for geometric sources.

\section{Conflict of Interests}

The authors declare that there is no conflict of interests regarding the publication of this paper.

\section{Acknowledgments}

This work was supported in part by the National Research Foundation for the Doctoral Program of Higher Education of China under Grant 20120005110007 and the NSFC under Grants 61271257 and 61379159. 


\section{References}

[1] Ö. Oyman and S. Sandhu, "A shannon-theoretic perspective on fading multihop networks," in Proceedings of the 40th Annual Conference on Information Sciences and Systems (CISS '06), pp. 525-530, Princeton, NJ, USA, March 2006.

[2] M. Sikora, J. N. Laneman, M. Haenggi, D. J. Costello Jr., and T. E. Fuja, "Bandwidth- and power-efficient routing in linear wireless networks," IEEE Transactions on Information Theory, vol. 52, no. 6, pp. 2624-2633, 2006.

[3] D. Rajan, "Power efficient delay allocation in multihop wireless networks," IEEE Transactions on Vehicular Technology, vol. 56, no. 4, pp. 1813-1825, 2007.

[4] M. Haenggi, "On routing in random rayleigh fading networks," IEEE Transactions on Wireless Communications, vol. 4, no. 4, pp. 1553-1562, 2005.

[5] M. Xie and M. Haenggi, "Towards an end-to-end delay analysis of wireless multihop networks," Ad Hoc Networks, vol. 7, no. 5, pp. 849-861, 2009.

[6] D. E. Wrege, E. W. Knightly, H. Zhang, and J. Liebeherr, "Deterministic delay bounds for VBR video in packet-switching networks: fundamental limits and practical trade-offs," IEEE/ACM Transactions on Networking, vol. 4, no. 3, pp. 352-362, 1996.

[7] E. W. Knightly and H. Zhang, "D-BIND: an accurate traffic model for providing QoS guarantees to VBR traffic," IEEE/ACM Transactions on Networking, vol. 5, no. 2, pp. 219-231, 1997.

[8] M. Xie and M. Haenggi, "A study of the correlations between channel and traffic statistics in multihop networks," IEEE Transactions on Vehicular Technology, vol. 56, no. 6 I, pp. 35503562, 2007.

[9] K. Stamatiou and M. Haenggi, "Delay characterization of multihop transmission in a poisson field of interference," IEEE/ACM Transactions on Networking, vol. 22, no. 6, pp. 1794-1807, 2014.

[10] D. Stoyan, W. S. Kendall, and J. Mecke, Stochastic Geometry and Its Applications, John Wiley \& Sons, 2nd edition, 1996.

[11] F. Baccelli, B. Blaszczyszyn, and P. Muhlethaler, "An aloha protocol for multi-hop mobile wireless networks," IEEE Transactions on Information Theory, vol. 52, no. 2, pp. 421-436, 2006.

[12] S. M. Ross, Stochastic Processes, John Wiley \& Sons, New York, NY, USA, 2nd edition, 1996. 

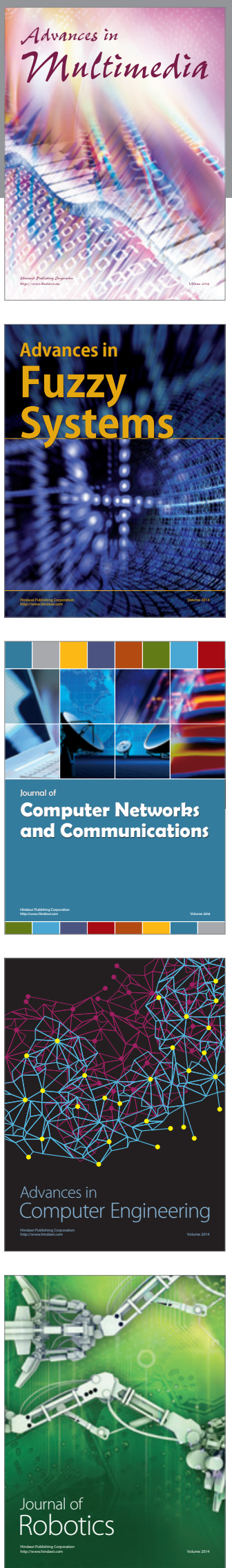

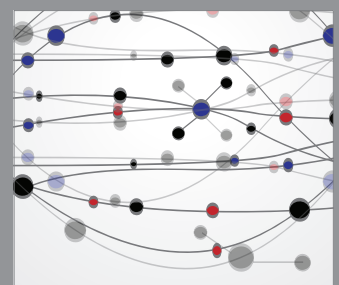

The Scientific World Journal
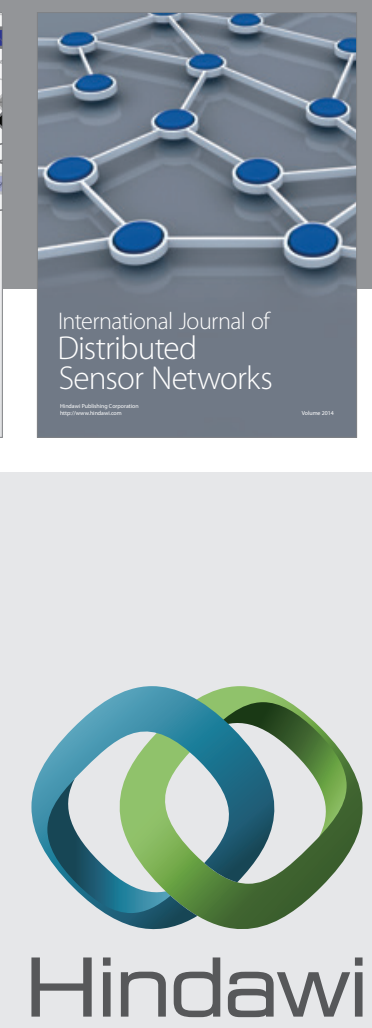

Submit your manuscripts at

http://www.hindawi.com
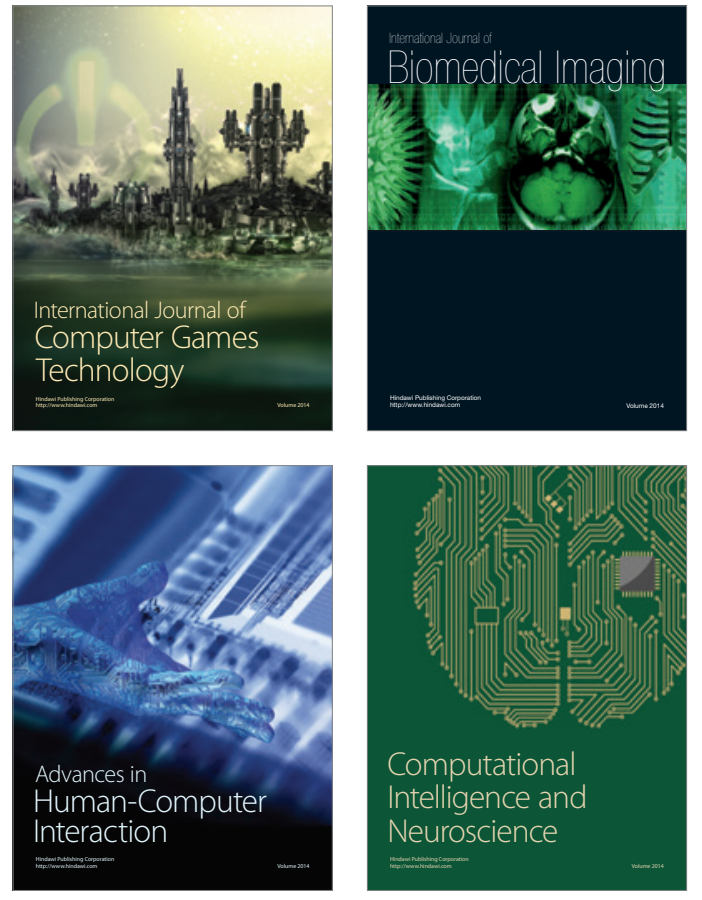
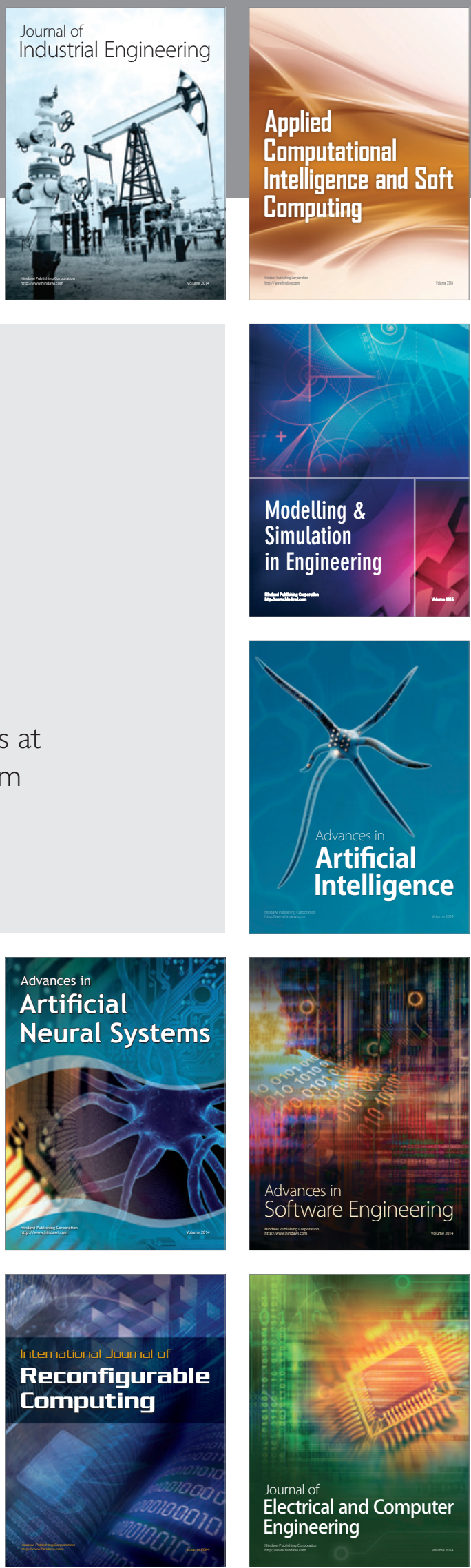